\title{
Energy Transfer Processes in InAs/GaAs Quantum Dot Bilayer Structure
}

\author{
M. Pieczarka ${ }^{a}$, A. Maryński ${ }^{a}$, P. Podemski ${ }^{a}$, J. Misiewicz ${ }^{a}$, P.D. Spencer ${ }^{b}$, \\ R. MURRAY ${ }^{b}$ AND G. SĘK ${ }^{a}$ \\ ${ }^{a}$ Laboratory for Optical Spectroscopy of Nanostructures, Department of Experimental Physics, \\ Wrocław University of Technology, Wybrzeże Wyspiańskiego 27, 50-370 Wrocław, Poland \\ ${ }^{b}$ Blackett Laboratory, Physics Department, Imperial College London, London, SW7 2AZ, United Kingdom
}

\begin{abstract}
We investigate double layer InAs/GaAs quantum dots grown in the Stransky-Krastanov mode by molecular beam epitaxy. The sample consists of two layers of InAs quantum dots separated by $10 \mathrm{~nm}$ thick GaAs layer, where the top quantum dot layer of an improved homogeneity is covered by an InGaAs cap. This configuration has allowed for the extension of the dots' emission to longer wavelengths. We probed the carrier transfer between the states confined in a double quantum well composed of InGaAs cap and the quantum dots wetting layer to the states in the quantum dots by means of photoluminescence excitation and photoreflectance spectroscopies. Efficient emission from quantum dots excited at the double quantum well ground state energy was observed. There is also presented a discussion on the carrier injection efficiency from the capping layer to the quantum dots.
\end{abstract}

DOI: 10.12693/APhysPolA.129.A-59

PACS: 78.67.Hc, 71.35.-y, 78.55.-m

\section{Introduction}

Semiconductor nanostructures based on III-V group elements are well known and widely investigated for over a decade [1]. Quantum dots (QDs) made of InAs and grown epitaxially on GaAs are one of the first well established technologically and extensively explored for applications in optoelectronic devices such as lasers, optical amplifiers or novel quantum devices like single photon sources [2]. The technology is well developed in all these applications for emission region below $1 \mu \mathrm{m}$, which is a typical energy range for QDs grown in $\mathrm{InAs} / \mathrm{GaAs}$ epitaxy. Nevertheless, achievement of longer emission wavelengths, to employ these QD devices in the optical telecommunication, is still challenging from the technological site. New sample designs and different growing conditions have to be implemented to fulfill this application domain. Indeed, the extension of QD emission to longer wavelengths is possible to realize by several growth techniques like epitaxy at low growth rates [3], alternate epitaxy of In, Ga and $\mathrm{As}_{2}$ layers [4], capping the QDs with a thin layer of InGaAs [5,6] or integrating the QDs within a quantum well (QW) of a lower bandgap than pure GaAs [7]. However, these approaches lead to difficulties in obtaining appropriate homogeneity of QDs for high device efficiency. For this reason, there is a necessity to establish a technique, where the long-wavelength emission joint with high QD homogeneity is obtained. Therefore, rather than using a single QD layer in the structure one can instead exploit the design of two closely stacked QD layers, allowing further control of the QD growth conditions. This kind of approach is beneficial, as the natural strain field induced by the QDs in the first layer allows growing more homogeneous and larger dots in the second layer $[8]$.

In this work we present spectroscopic studies on a structure designed in this alternative approach, where the bilayer QDs based on InAs grown on GaAs emits at wavelengths as long as $1.2 \mu \mathrm{m}$ at cryogenic temperature. We performed optical spectroscopy measurements in order to investigate the energy structure and energy transfer between different parts of the system and observed efficient QD excitation via the capping layer. The effectiveness of this excitation of the QD layer is discussed.

\section{Experimental details}

The investigated structure consists of double epitaxial InAs QD layer grown on a GaAs substrate by molecular beam epitaxy (MBE) in the Stransky-Krastanov mode. The scheme of the full structure is presented in Fig. 1a. The bottom seed-layer was grown at $470{ }^{\circ} \mathrm{C}$ with a $2 \mathrm{D}$ 3D transition at approximately 1.7 ML of InAs deposition. This layer was covered with $10 \mathrm{~nm}$ of GaAs, providing an inhomogeneous strain field for the growth of the next QD layer. The top QD layer was deposited at the same growth rate and conditions, nevertheless the 2D$3 \mathrm{D}$ growth transition was observed at earlier deposition stage, because of the strain stacking effect of the QDs, which led to creation of more homogeneous and larger dots of low density. To extend the emission layer to longer wavelengths, the upper layer was covered with $4 \mathrm{~nm}$ layer of $\operatorname{In}_{0.25} \mathrm{Ga}_{0.75}$ As to suppress the strain-enhanced indium and gallium intermixing in the top-layer QDs and then capped with $100 \mathrm{~nm}$ GaAs. Further details on the growth procedure can be found elsewhere [9].

We investigated the optical properties of the QD bilayer structure by means of absorption- and emissiontype of spectroscopy. The noninvasive, absorption-like technique was utilized, namely photoreflectance (PR) within a bright configuration setup allowing for the detection of normalized changes in the reflectivity coefficient $(\Delta R / R)$ with high sensitivity [10]. The emission properties of the QDs were investigated in a 

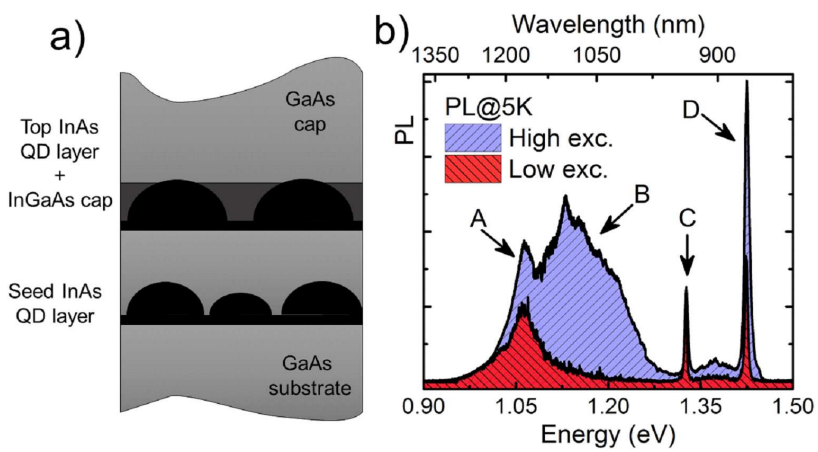

Fig. 1. (a) Schematic representation of the investigated double layer QD sample. (b) Macrophotoluminescence under nonresonant excitation recorded at two different excitation power densities 0.03 and $3 \mathrm{~kW} / \mathrm{cm}^{2}$. Emission features are marked as: A - top QD layer capped with InGaAs layer, $\mathrm{B}$ - bottom QD seed layer, C - InGaAs capping layer, D - bulk GaAs emission.

photoluminescence (PL) under nonresonant excitation at wavelength of $640 \mathrm{~nm}(1.937 \mathrm{eV})$ and photoluminescence excitation (PLE) experiments. The PLE system was equipped with a tunable external-cavity semiconductor diode laser with narrow linewidth $(\ll 1 \mu \mathrm{eV})$, combined with a customized automatic power-stabilizing system to ensure the same laser power density for each used excitation wavelength. In all experiments, the sample was mounted in a helium-flow microscopy cryostat to perform measurements at cryogenic temperatures. The spectra were collected and analyzed with a half-meter-focallength monochromator, equipped with a nitrogen-cooled InGaAs linear array.

\section{Results}

The low temperature photoluminescence spectra of the QD ensemble obtained at nonresonant excitation at two power densities are presented in Fig. 1b. At low excitation density one can observe three distinct emission peaks. The lowest energy one, denoted as A, is the toplayer QD emission. Further, the strong and narrow peak at $1.32 \mathrm{eV}$ ( $\mathrm{C}$ in the figure) is observed and is attributed to the ground state of the InGaAs cap layer coupled to the InAs wetting layer (WL) of the top QDs, effectively being an asymmetric double quantum well (DQW). The high energy emission peak is simply the emission from the bulk GaAs and is marked as D. At higher excitation density, a broadband emission emerges at the high energy side of the top layer QDs peak (B in Fig. 1). This broad luminescence can be attributed to more inhomogeneous bottom seed layer of QDs. Seed layer QDs are smaller than those in the top layer, resulting in a blueshifted emission energy. The difference between the two QD layers is that the top layer QDs are the energy ground state of the system, therefore one can observe luminescence even at low carrier densities, because of the efficient feeding with carriers. The inequality of luminescence signal level of top and bottom QDs is also partly due to the suppressed feeding of the bottom layer QDs, as they are expected to be coupled to the top layer $[9,11]$. Higher excitation densities overcome this effect due to just filling the higher energy states of the entire system, and one can observe broad luminescence from the seed layer QDs in addition to the one from the top layer. It should be noted that the excited state of the upper layer QDs is expected to be $60-80 \mathrm{meV}$ above the ground state, based on our 8 -band $\boldsymbol{k} \cdot \boldsymbol{p}$ modelling [12] for a realistic geometry and homogeneous In distribution within the QD. These states might overlap with the emission from the lower QDs, however further measurements, e.g. single dot spectroscopy are necessary to distinguish between them, which is beyond the scope of this work.

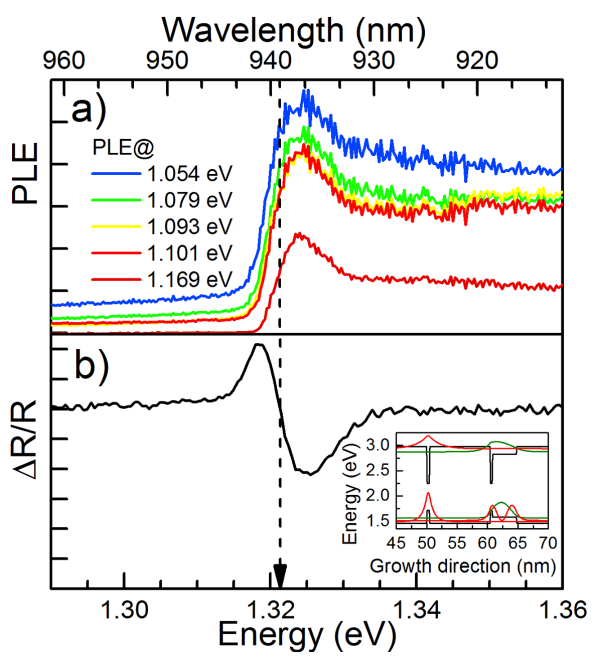

Fig. 2. (a) PLE spectra for various QD emission energies, excited around the ground state energy of the InGaAs cap layer. (b) PR spectrum showing the ground state transition in the InGaAs cap layer and calculated conduction and valence band edges together with probability densities of the confined states in the structure (inset). The ground states are marked by green lines.

The efficient carrier injection in the QD structures is crucial to obtain high performance quantum devices. To investigate these properties of the bilayer QD structure, we performed PLE measurements on the ensemble of QDs. The excitation energy was tuned in the range around the ground state of the InGaAs/InAs DQW and the power density was kept constant $\left(P=10 \mathrm{~kW} / \mathrm{cm}^{2}\right)$. The PLE spectra for different QD emission energies are plotted in Fig. 2a. Evident and steep increase of PLE signal is observed at $1.322 \mathrm{eV}$ for all QDs' emission lines. This energy value is in an excellent agreement with the DQW ground state energy obtained from PR experiment, presented in Fig. 2b, where one can observe a distinct PR resonance corresponding to this transition. Furthermore, we examined the energy structure of this system, performing 8-band $\boldsymbol{k} \cdot \boldsymbol{p}$ calculations [12]. We calculated the energy states in the full structure, taking into consideration both WLs and the capping layer made of InGaAs. Obtained results confirmed a single confined electron state and the ground hole state within 
the InGaAs/InAs DQW layer with the transition energy of $1.322 \mathrm{eV}$. The calculated probability densities of the confined electron and hole states in the structure are presented in the inset of Fig. 2b.

The shape of the PLE curve is similar for all emission energies, indicating similar injection mechanism to the QDs. There is a well-defined maximum in the PLE signal having similar width as the feature in the PR spectrum. This PLE maximum is attributed to the feeding of the QD state via ground state excitonic transition in the InGaAs QW layer. At higher excitation energies, one can observe a characteristic decrease and a plateau of the PLE signal. This feature is due to the well-known density of states within the 2D QW, which is formed from the InGaAs/InAs DQW, where only one state is confined. Similar features can be observed in dot-in-a-well (DWELL) structures [13].

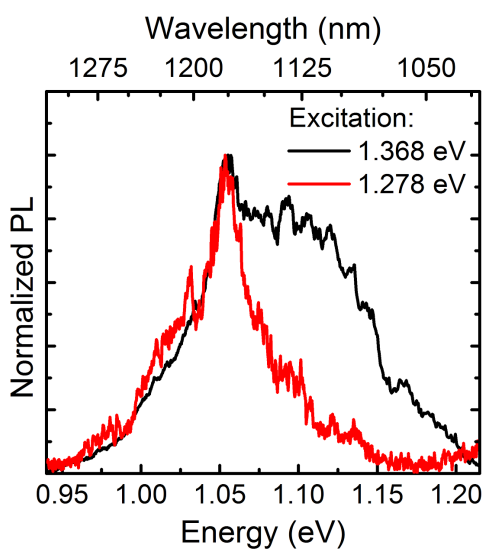

Fig. 3. Normalized PL intensity at different excitation energies, obtained for the same excitation power density of $10 \mathrm{~kW} / \mathrm{cm}^{2}$.

Further, we analyzed the emission spectra, when the excitation energy was tuned below the InGaAs/InAs DQW ground state. In this energy range the absorption of the exciting laser is inefficient, so the number of photo-excited carriers is dramatically lower in comparison to the excitation energy above the InGaAs layer confined state. To compare the spectra at these two conditions, we present normalized PL (Fig. 3) recorded at different excitation energies: below the ground state of DQW $(1.278 \mathrm{eV})$ and above $(1.368 \mathrm{eV})$. The emission spectrum for the excitation above the cap layer state is similar to the nonresonant scheme at high excitation, where both QD families are efficiently fed and one can observe strong luminescence. On the other hand, at lower energy excitation, weak signal originating only from the upper QD layer is present. The top layer QD luminescence is about 5 times weaker in comparison to the excitation via InGaAs/InAs DQW state. It is evidently caused by the lower electron-hole pairs number at this excitation energy. Moreover, this reminds a similar situation at nonresonant excitation and low power densities, where small carrier concentration fed only the top-layer
QDs. Concluding, it proves the significance of the InGaAs layer as an efficient channel for carrier capture and injection into the active region of the sample, composed of two QD layers.

\section{Conclusions}

We investigated a bilayer QD structure emitting at extended wavelength of $1.2 \mu \mathrm{m}$ at cryogenic temperatures. The upper QD layer has shown a higher homogeneity in comparison to lower seed layer of the QDs, even though both layers were grown at similar conditions. We observed an efficient feeding of the QD states via the ground state confined in the DQW composed from InGaAs capping layer and InAs WL. We confronted the results with theoretical values obtained from $\boldsymbol{k} \cdot \boldsymbol{p}$ modelling. We predict this nanostructure design as excellent for application in QD lasers, where the carrier capturing and efficient feeding of the active material is of great importance.

\section{Acknowledgments}

Authors would like to thank $€$. Dusanowski for useful results' discussion. Financial support from the Polish Ministry of Science and Higher Education within the "Diamond Grant" project No. DI2012 008642 is gratefully acknowledged.

\section{References}

[1] P. Michler, Single Semiconductor Quantum Dots, Springer, Heidelberg 2009.

[2] E. Moreau, I. Robert, J.M. Gérard, I. Abram, L. Manin, V. Thierry-Mieg, Appl. Phys. Lett. 79, 2865 (2001).

[3] R. Murray, D. Childs, S. Malik, P. Siverns, C. Roberts, J.-M. Hartmann, P. Stavrinou, Jpn. J. Appl. Phys. 38, 528 (1999).

[4] R.P. Mirin, J.P. Ibbetson, K. Nishi, A.C. Gossard, J.E. Bowers, Appl. Phys. Lett. 67, 3795 (1995).

[5] K. Nishi, H. Saito, S. Sugou, J.-S. Lee, Appl. Phys. Lett. 74, 1111 (1999).

[6] R. Songmuang, S. Kiravittaya, M. Sawadsaringkarn, S. Panyakeow, O.G. Schmidt, J. Cryst. Growth 251, 166 (2003).

[7] V.M. Ustinov, N.A. Maleev, A.E. Zhukov, A.R. Kovsh, A.Y. Egorov, A.V. Lunev, B.V. Volovik, I.L. Krestnikov, Y.G. Musikhin, N.A. Bert, P.S. Kop'ev, Z.I. Alferov, N.N. Ledentsov, D. Bimberg, Appl. Phys. Lett. 74, 2815 (1999).

[8] Q. Xie, A. Madhukar, P. Chen, N.P. Kobayashi, Phys. Rev. Lett. 75, 2542 (1995).

[9] M. Majid, D. Childs, H. Shahid, S. Chen, K. Kennedy, R.J. Airey, R.A. Hogg, E. Clarke, P. Howe, P.D. Spencer, R. Murray, IEEE J. Sel. Top. Quantum Electron. 17, 1334 (2011).

[10] J. Misiewicz, G. Sęk, R. Kudrawiec, P. Sitarek, Thin Solid Films 450, 14 (2004).

[11] E.C. Le Ru, A.J. Bennett, C. Roberts, R. Murray, J. Appl. Phys. 91, 1365 (2002).

[12] www.nextnano.com, Nextnano GmbH

[13] R. Chen, H.Y. Liu, H.D. Sun, J. Appl. Phys. 107, 013513 (2010). 